\title{
VIABILIDADE ECONÔMICA EM SISTEMA AGROFLORESTAL NO MUNICÍPIO DE SANTA IZABEL DO PARÁ, PA
}

Alinne Santos Silva, Rocha ${ }^{1}$, Stefany Castro Brito ${ }^{2}$, Ismael Matos da Silva ${ }^{3}$, Manoel Tavares de Paula ${ }^{3}$ e Bruna Stefanny das Neves de Sousa ${ }^{4}$

1 Mestranda do Programa de Pós-graduação em Recursos Florestais-ESALQ- USP

2. Engenheira Florestal da Universidade do Estado do Pará

3 Professores doutores da Universidade do Estado do Pará(dpaulamt@hotmail.com) 4 Mestranda do Programa de Pós-graduação em Ciências Ambientais da Universidade do Estado do Pará

\section{Recebido em: 06/04/2018 - Aprovado em: 10/06/2018 - Publicado em: 20/06/2018} DOI: 10.18677/EnciBio_2018A83

\begin{abstract}
RESUMO
Os Sistemas Agroflorestais (SAF) constituem-se em alternativa de uso da terra para aliar a estabilidade do ecossistema visando à eficiência e otimização de recursos naturais na produção de forma integrada e sustentada. O objetivo desse trabalho foi avaliar a viabilidade econômica de um sistema agroflorestal localizado no município de Santa Izabel no Pará. O sistema agroflorestal é formado por espécies agrícolas: mandioca (Manihot esculenta Crantz) e cupuaçu (Theobroma grandiflorum (Willd. ex Spreng.) Schum.), e com a espécie florestal paricá (Schizolobium amazonicum (Huber ex Ducke). A rentabilidade econômica do sistema foi mensurada utilizandose os seguintes parâmetros de avaliação, Valor Presente Líquido (VPL), a Taxa Interna de Retorno (TIR), a Razão Benefício/Custo (RB/C) e o Ponto de Nivelamento (PN). Para os cálculos foi escolhida uma taxa de 2,5\%. O estudo demonstrou que o sistema agroflorestal apresentou rentabilidade econômica positiva, e que pode ser adotado por pequenos produtores. Assim, o sistema agroflorestal é economicamente viável para pequenos produtores rurais e agricultores familiares trabalhando nas mesmas condições apresentadas neste estudo.
\end{abstract}

PALAVRAS-CHAVE: Avaliação econômica. Amazônia. Produtor familiar

\section{ECONOMIC VIABILITY IN AGROFLORESTAL SYSTEMS IN THE MUNICIPALITY OF SANTA IZABEL DO PARÁ, PA}

\begin{abstract}
Agroforestry Systems (SAFs) constitute an alternative land use to combine the stability of the ecosystem aiming at the efficiency and optimization of natural resources in production in an integrated and sustained way. This work aimed to evaluate the economic viability of an agroforestry system, located in the municipality of Santa Izabel, in the state of Pará. The system is composed of agricultural species: cassava (Manihot esculenta Crantz) and cupussu (Theobroma grandiflorum (Willd. Ex Spreng. Schum.), having as parica forest species (Schizolobium amazonicum Ducke). The economic profitability of the system was measured using the Net
\end{abstract}


Present Value (NPV), the Internal Rate of Return (IIR), the Benefit / Cost Ratio $(\mathrm{RB} / \mathrm{C})$ and the Leveling Point (PN) as the financial indicators. For the analysis of the updated net present value, a rate of $2,5 \%$ per annum was chosen. The results showed that the SAF studied presented a positive economic profitability, and this model can be adopted by small producers in the region, as well as being able to be used as a model in the planning of other SAF present in other locations, with the same species studied in the work in question.

KEYWORDS: Economic evaluation. Amazon. Family producer.

\section{INTRODUÇÃO}

O processo de desmatamento na Amazônia brasileira foi resultado da abertura de estradas que culminou com a exploração madeireira e o desenvolvimento de pastagens, ao longo dos anos. Os migrantes vindos do Sul e Sudeste do Brasil se estabeleceram legalmente em terras da Floresta Amazônica, extraindo madeira, que era comercializada para financiar a implantação de suas atividades econômicas (YAMADA : GHOLZ, 2002).

Para Reydon (2011) o desmatamento da Amazônia é fruto da continuidade da tradicional forma de expansão da fronteira agrícola brasileira, que, em geral, costuma ocorrer por meio da ocupação de terras virgens (privadas ou públicas), da extração de madeira de lei, a instalação da pecuária e, modernização da agropecuária. Essas atividades econômicas exercem o papel de gerar renda e legitimar a ocupação dos novos proprietários no curto prazo, quase sem necessidade de recursos. No longo prazo, as terras ou permanecem com pecuária mais intensiva, ou, se existir demanda, serão convertidas para grãos ou outra atividade econômica.

O esgotamento dos solos, a diminuição dos estoques de recursos florestais, alterações microclimáticas, alterações dos mananciais, entre outros acontecimentos, tem conduzido a sociedade a tomar medidas e ações direcionadas para sua preservação, no sentido de operar ações de plantio e desenvolvimento, de modo a assegurar, não apenas sua perpetuação, mas sua utilização comercial para os mais variados fins, sem que afete o estoque natural da floresta (FALESI ; GALEÃO, 2004).

Os sistemas agroflorestais podem ser definidos como sendo a modalidade de uso integrado da terra para fins de produção florestal, agrícola e pecuário (DUBOIS, 1996). Os sistemas agroflorestais são vistos como alternativa promissora para propriedades rurais dos países em desenvolvimento. Pela integração da floresta com culturas agrícolas e com a pecuária, esse sistema oferece alternativa para os problemas da baixa produtividade, de escassez de alimentos e da degradação ambiental generalizada (DUBOIS, 1996).

No setor agropecuário, os sistemas agroflorestais são uma das alternativas indicadas para serem implementadas na Amazônia como forma de mitigação das emissões dos gases do efeito estufa (GEE), com vista em reduzir os efeitos do aquecimento global sobre a produção vegetal.

As espécies utilizadas para formação do SAF pelos produtores familiares do município de Santa Izabel do Pará são o paricá, a mandioca e o cupuaçu. O paricá Schizolobium parahyba var. amazonicum (Huber x Ducke) Barneby se adapta bem em sistemas agroflorestais mais adensados ou em plantios puros, apresenta rápido crescimento em altura, boa adaptação às diferentes condições edafoclimáticas da ENCICLOPÉDIA BIOSFERA, Centro Científico Conhecer - Goiânia, v.15 n.27; p.156 
região, sendo considerado uma alternativa regional, para suprir a demanda de matéria-prima florestal, bem como para recomposição de áreas alteradas (SILVEIRA, 2014; CORDEIRO et al., 2015).

A cultura da mandioca (Manihot esculenta Crantz) é uma espécie de grande importância econômica no território Brasileiro, apresenta-se como um interessante componente em sistemas agroflorestais, por constituir-se como a base alimentar para aproximadamente 500 milhões de pessoas no mundo, sendo importante fonte de energia alimentar, além de ser utilizada no arraçoamento animal e como matériaprima para diversos produtos agroindustrializados (FAO, 2013; GOMES \& CARDOSO, 2014).

O cupuaçu (Theobroma grandiflorum (Willd. ex Spreng.) Schum.) juntamente com o cacau (Theobroma cacao L.) é uma das principais culturas perenes na rentabilidade dos sistema agroflorestal em Tomé-Açu (KONAGANO et al., 2016). A produção atual de cupuaçu provém, basicamente, de plantios comerciais, estimados em mais de 20 mil hectares distribuídos no Pará (11.867 ha), Amazonas, Rondônia e Acre, principalmente (ALVES et al., 2014).

O objetivo do presente estudo foi avaliar a viabilidade econômica de um Sistema Agroflorestal (SAF) formado com as espécies paricá, mandioca e cupuaçu no município de Santa Isabel do Pará. Estado do Pará, Brasil.

\section{MATERIAL E MÉTODOS}

\section{ÁREA DE ESTUDO}

O presente estudo foi realizado no município de Santa Izabel do Pará (Figura 1), localizado na região de integração do Guamá, zona fisiográfica Bragantina, no nordeste paraense, mesorregião metropolitana de Belém e micro região Castanhal, situado entre as coordenadas geográficas $01^{\circ} 17^{\prime} 27^{\prime \prime}$ e $01^{\circ} 32^{\prime} 54^{\prime \prime}$ de latitude sul e $47^{\circ} 59^{\prime} 38^{\prime \prime}$ de longitude oeste de Greenwich, distante a $40 \mathrm{Km}$ da capital Belém (PREFEITURA SANTA IZABEL, 2016). Sua população estimada no ano de 2017 é de 68.836 pessoas (IBGE, 2017).

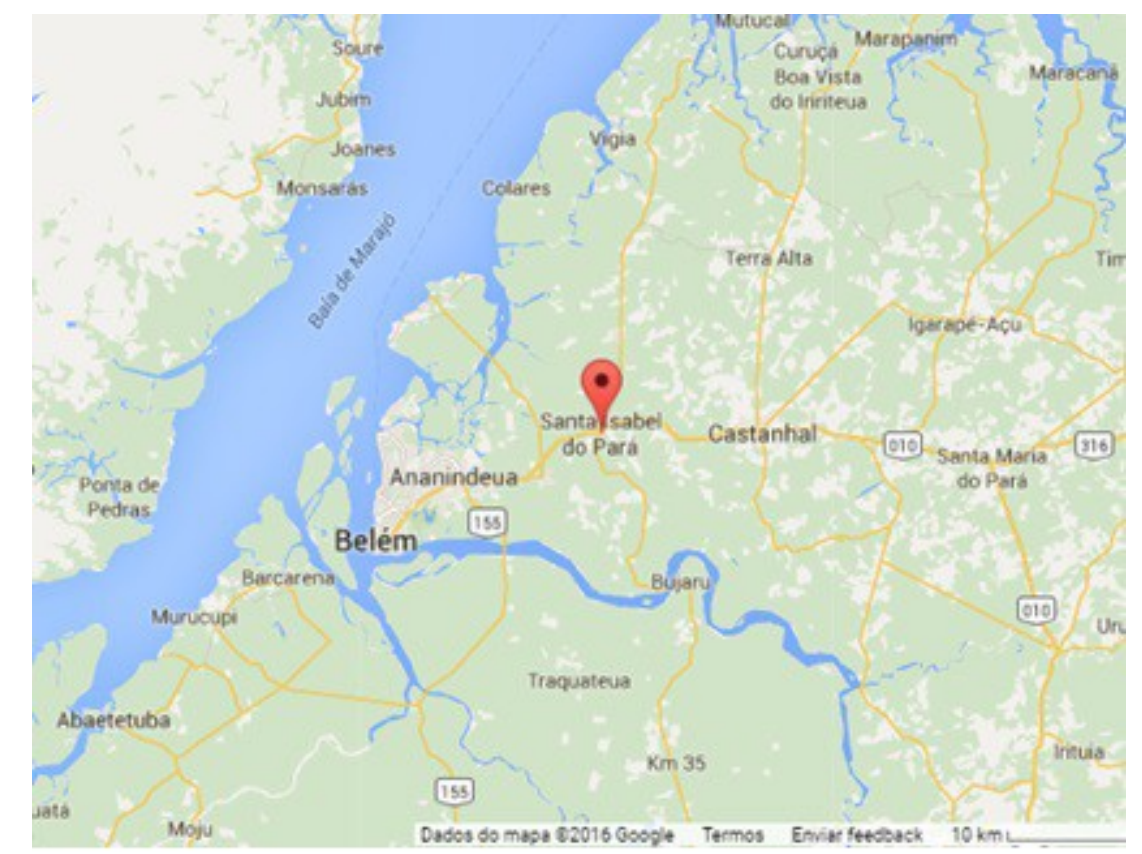

FIGURA 1 - Localização do município de Santa Izabel do Pará 
De acordo, com Pires et al., (2006) o solo da região é constituído pelos seguintes tipos: Concrecionários lateríticos indiscriminados distróficos; Latossolo Amarelo distrófico, textura média; Concrecionários Lateríticos e Areias Quartezosas. a vegetação predominante são: Florestas Secundárias, decorrentes da recomposição natural da vegetação, quando a Floresta Primária foi removida pelo desmatamento. Apresenta clima megatérmico úmido, com temperatura elevada, estando a média mensal em torno de $25^{\circ} \mathrm{C}$, tendo os meses de outubro, novembro e dezembro os mais quentes, com máximas entre $32^{\circ} \mathrm{C}$ e $34^{\circ} \mathrm{C}$ e mínima entre $20^{\circ} \mathrm{C}$ e $22^{\circ} \mathrm{C}$, também, caracterizado por inverno quente, com precipitações em torno de $2.350 \mathrm{~m} . a n o^{-1}$, concentradas de janeiro a junho, com maior escassez em setembro; sua umidade relativa do ar está em torno de $85 \%$.

\section{ESTRUTURA DO PLANTIO}

A área utilizada para estudo contendo a aplicação do SAF com paricá, cupuaçu e mandioca (Figura 2) constitui-se de 1 hectare da área de produção dos agricultores familiares pertencentes ao município de Santa Izabel no Estado do Pará.

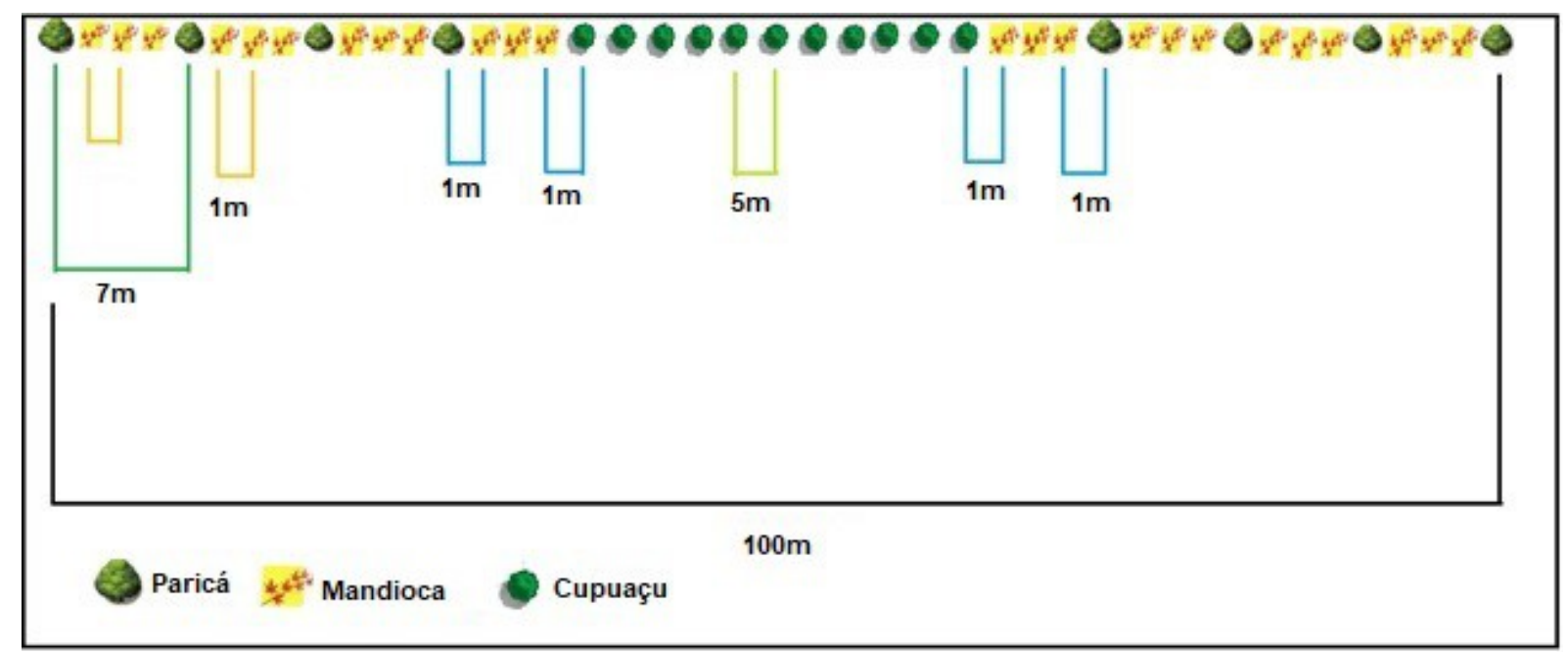

FIGURA 2 - Esquema de uma da lateral superior do SAF para ser implantação de Fonte: autores paricá, mandioca e cupuaçu em 1 ha.

Com relação à implantação espacial das culturas estudadas em campo, foram diagnosticadas a partir de informações de dados com os agricultores do local e implantadas da seguinte forma (Tabela 1): $4.200 \mathrm{~m}^{2}$ para a plantação de Paricá, subdividindo em duas áreas na parte lateral esquerda e direita, como mostra a figura 2; cada área com $2.100 \mathrm{~m}^{2}$. Cada uma dessas áreas possui quatro fileiras, e cada fileira com 15 mudas de paricá, com espaçamento $7 \times 7 \mathrm{~m}$. No total são 120 mudas de paricá, sendo 60 mudas em cada subárea.

As plantas de mandioca ocupam $400 \mathrm{~m}^{2}$ da área subdividida em duas de $200 \mathrm{~m}^{2}$ ao lado das áreas de paricá, e três fileiras de mandioca entre cada muda de paricá, formando uma área de $200 \mathrm{~m}^{2}$. O total de área de mandioca compreende a $1.600 \mathrm{~m}^{2}$ (sendo $1.200 \mathrm{~m}^{2}$ o tamanho da área com as mudas de mandioca e paricá). O espaçamento utilizado é de $1 \times 1 \mathrm{~m}$. No total foram utilizadas 2.400 estacassementes de mandioca, sendo 100 estacas-sementes em cada fileira. 
A implantação dos cupuaçuzeiros foi realizada em uma área de $5.000 \mathrm{~m}^{2}$ no centro da área e entre os plantios de mandioca. O espaçamento utilizado para o cupuaçu foi de $5 \times 5 \mathrm{~m}$. Foram utilizadas onze fileiras para o plantio, sendo cada uma das fileiras com 21 mudas de cupuaçu, totalizando 231 mudas da espécie.

Com relação à plantação do cupuaçuzeiro, temos uma área de 5.000 metros no centro, entre uma das áreas de plantação de mandioca. O espaçamento utilizado para o cupuaçu foi de $5 \times 5$. Foram utilizadas 11 fileiras para plantar o cupuaçu, e cada uma das fileiras com 21 mudas de cupuaçu, totalizando 231 mudas da espécie.

A área restante para completar um hectare da área para o SAF é a do espaçamento do paricá para a plantação de mandioca, da mandioca para a plantação de cupuaçu. Isso nas duas áreas totalizando uma área de 400 metros, como pode ver em destaque na figura 2.

TABELA 1- Espécies, número de plantas e espaçamento de plantio no SAF, para uma área de 1 hectare (ha) Santa Izabel-PA.

\section{Espécies \\ Número de Plantas para Espaçamento $(\mathrm{m})$ a área de 1 ha}

\begin{tabular}{lcc}
\hline Paricá & 120 & $7,00 \times 7,00$ \\
\hline Mandioca & 2.400 & $1,00 \times 1,00$ \\
\hline Cupuaçu & 231 & $5,00 \times 5,00$ \\
\hline
\end{tabular}

Fonte: Dados de pesquisa

\section{Mortalidade das espécies}

Como é mostrado na Tabela 2, levando-se em consideração a possibilidade de ocorrência de danos ou incidentes, no transporte das mudas e estacas até a área de cultivo, considerou-se uma mortalidade a $10 \%$ para o paricá, a mandioca e o cupuaçu. A mesma porcentagem também foi utilizada para a mortalidade durante a colheita, para todas as espécies em um hectare do SAF.

TABELA 2 - Espécies, número de plantas a serem plantadas na área de 1 ha, o número de plantas a mais com a mortalidade à $10 \%$, e total de mudas

\begin{tabular}{lcclccc}
\hline Espécies & $\begin{array}{l}\text { Número de } \\
\text { Plantas } \\
\text { área de 1 ha }\end{array}$ & $\begin{array}{l}\mathbf{N}^{\circ} \text { de plantas a } \\
\text { mais com a } \\
\text { taxa } \\
\text { mortalidade } \\
\text { das mudas }\end{array}$ & $\begin{array}{l}\text { Totais } \\
\text { compradas }\end{array}$ & $\begin{array}{l}\mathbf{N}^{\circ} \text { de plantas } \\
\text { colhidas com } \\
\text { a taxa de } \\
\text { mortalidade da } \\
\text { colheita }\end{array}$ \\
\hline Paricá & 120 & 12 & 132 & 108 \\
\hline Mandioca & 2.400 & 240 & 2.640 & 2.160 \\
\hline Cupuaçu & 231 & 23 & 254 & 208 \\
\hline
\end{tabular}

Fonte: Dados da pesquisa

$\mathrm{Na}$ colheita das toras, frutos e mandioca, também pode haver a mortalidade. Assim, outra porcentagem em cima do número de mudas e tubetes plantados.

\section{Compra de mudas e estacas}

$\mathrm{Na}$ Tabela 3, têm-se os custos de mudas (paricá e cupuaçu) e estacas vegetativas (mandioca) que constituem o sistema agroflorestal estudado. As mudas de paricá e cupuaçu foram compradas na AIMEX (Associação das Indústrias 
Exportadoras de Madeira do Pará), que também está presente na ASFLORA (Instituto Amigos da Floresta Amazônica), no laboratório de mudas e sementes, no município de Benevides, a 15 quilômetros $(\mathrm{km})$ de Santa Izabel. Já as estacas de mandioca para o plantio, as mesmas foram compradas de outros produtores da região.

TABELA 3 - Espécies, preço, total de mudas e estacas compradas e total de preços.

\begin{tabular}{|c|c|c|c|}
\hline Espécies & Preço (R\$) & $\begin{array}{l}\text { Total de mudas e } \\
\text { estacas } \\
\text { compradas }\end{array}$ & $\begin{array}{l}\text { Total do preço } \\
\text { (R\$) }\end{array}$ \\
\hline Paricá & 2,00 (tubete) & 132 & 264,00 \\
\hline Mandioca & 0,50 (estaca) & 2.640 & $1.320,00$ \\
\hline Cupuaçu & 2,80 (tubete) & 254 & 711,20 \\
\hline
\end{tabular}

Fonte: Dados de pesquisa

Venda da produção do SAF

O valor dos preços de venda dos produtos obtidos no sistema agroflorestal estudado, encontra-se na tabela 4, juntamente com suas respectivas unidades de comercialização.

TABELA 4 - Espécies, unidade de medida e preço de venda

\begin{tabular}{lcc}
\hline \multicolumn{1}{c}{ Espécies } & Unidade & Preço de venda (R\$) \\
\hline Paricá - madeira & tora & 50,00 \\
\hline Mandioca - raiz & $\mathrm{kg}$ & 0,50 \\
\hline Cupuaçu - fruto & $\mathrm{kg}$ & $0,60^{*}$ \\
\hline
\end{tabular}

Fonte: Dados da pesquisa

*O peso do fruto do cupuaçu foi estimado em $1,5 \mathrm{~kg}$, assim seu valor foi para $0,90 \mathrm{o}$ $\mathrm{kg}$ e meio.

A produção de madeira em tora será destinada ao comercio nacional para diferentes estados. A fruta e mandioca serão comercializadas no estado do Pará.

A produção de madeira em tora será destinada ao comercio nacional para diferentes estados. Os frutos dos cupuaçuzeiros e as raízes da mandioca serão comercializadas no Estado do Pará. Para análise dos valores econômicos utilizaramse os indicadores financeiros, descritos a seguir:

\section{MODELO DE ANÁLISE}

\section{Valor Presente Líquido - VPL}

É a diferença entre o valor investido e o valor regatado ao fim do investimento, trazidos ao valor presente. Se VPL for positivo, então o valor investido será recuperado e haverá um ganho. Se VPL for zero significa que aplicar ou não fará diferença. Agora se VPL for negativo significa que o investidor estará resgatando um valor menor que o valor investido, então não se deve aplicar neste investimento (SILVA ; FARIAS, 2015; GONÇALVES et al., 2017). 
$\mathrm{VPL}=\sum_{t=0}^{n}\left(\frac{R_{t}-C_{t}}{(1+i)^{t}}\right)$

Onde:

$\mathrm{R}_{\mathrm{t}}=$ fluxo de receita do projeto no ano $\mathrm{t}$;

$\mathrm{C}_{\mathrm{t}}=$ fluxo de custo do projeto no ano $\mathrm{t}$;

$\mathrm{i}=$ taxa de juros de longo prazo, em \%;

$\mathrm{n}=$ número de anos do projeto $(\mathrm{t}=1,2, \ldots, \mathrm{n})$.

\section{Taxa Interna de Retorno - TIR}

De acordo com De Paula et al. (2014) a Taxa Interna de Retorno (TIR) é a taxa que produz um VPL igual a zero. Considera-se atraente o projeto que apresentar uma TIR maior ou igual à TMA. Apesar de ser um dos métodos preferidos na análise de projetos, são necessários alguns cuidados especiais em sua utilização. Mesmo não sendo um dos melhores indicadores, a TIR é uma das formas de avaliação de projetos mais utilizadas no meio empresarial.

$\sum_{t=0}^{n} \operatorname{Re}$ ceita $_{t}{ }^{*}(1+\text { Tir })^{-t}=\sum_{t=0}^{n}$ Custo $_{t}{ }^{*}(1+\text { Tir })^{-t}$

em que:

Tir = taxa interna de retorno; e

$T$ = número de anos do projeto $(t=1, \ldots, n)$.

Relação Benefício/Custo - B/C

A Relação Benefício-Custo $(R B / C)$ é um indicador de eficiência econômica receita

financeira por sugerir o retorno dos investimentos a partir da relação entre a unidades de

total e as despesas efetuadas para viabilizá-la, ou seja, indica quantas

capital recebido como benefícios são obtidas para cada unidade de capital investido. (PALHETA et al., 2014). De acordo com esta medida de efetividade econômica, aceitam-se os projetos que apresentarem relação B/C maior do que 1 ; dado que os mesmos revelam possibilidades de produzirem benefícios em excesso aos custos (GONÇALVES et al., 2017).

Para efeito de análise importa que este índice seja maior que zero, uma vez que este resultado indica em quanto às receitas superam os custos. Por exemplo, em uma $B / C$ igual a 2,5 significa que para cada $R \$ 1,00$ (um real) investido (custo), o retorno bruto é de 2,5 reais em receita. $O$ índice é calculado por meio da seguinte fórmula (DE PAULA, 2014).

Onde:

$$
R_{B / C}=(V B(i) / V C(i))
$$

$\mathrm{VB}(\mathrm{i})=$ valor atual dos fluxos de benefícios à taxa de desconto i; e, $\mathrm{VC}(\mathrm{i})=$ valor atual dos custos à taxa de desconto $\mathrm{i}$. 


\section{Ponto de Equilíbrio - PE (ou ponto de nivelamento - PN)}

$O$ ponto de equilíbrio $(P E)$ é um indicador de curto prazo que revela o produto mínimo necessário para que as receitas se igualem com os custos, ou seja, este indicador mostra qual a quantidade mínima que o produtor precisa produzir para empatar as receitas com os custos. O ponto de equilíbrio é importante uma vez que estabelece o limite mínimo a ser produzido para evitar prejuízos com a atividade econômica. Para obtê-lo faz-se uso da seguinte equação matemática (DE PAULA, 2014).

Onde:

$$
\mathrm{PE}=1 /(\mathrm{RB} / \mathrm{C})
$$

PE: Ponto de Equilíbrio

$\mathrm{RB} / \mathrm{C}$ : Relação Benefício Custo

\section{Análise de sensibilidade}

Para verificar a capacidade do SAF em suportar choques externos à produção, tais como mudanças nos preços de venda e de aumento nos custos, foram simulados os seguintes cenários de análise de sensibilidade:

(2) redução nas receitas na ordem de $10 \%, 20 \%$ e $30 \%$; (1) aumento dos custos de produção na ordem de $10 \%, 20 \%$ e $30 \%$;

\section{RESULTADOS E DISCUSSÃO}

A pesquisa foi direcionada à agricultura familiar, para isto considerou-se o valor de $2,5 \%$ a.a, como taxa de desconto básica em conformidade com a taxa efetiva de juros estabelecida pelo Banco da Amazônia (BASA, 2018). O preço da terra utilizado foi estimado do resultado da média do preço mínimo e médio da terra nua encontrada disponibilizada pelo Instituto do Homem e Meio Ambiente da Amazônia - IMAZON (BRITO, 2015).

A premissa de permanência do SAF em campo foi de oito anos, com as culturas de Paricá, Mandioca e Cupuaçu, sendo a previsão de plantio para a mandioca de até o quinto ano, por ser uma cultura anual, colhida todo o ano.

O cupuaçu será colhido a partir do quarto ano, quando começa a produzir frutos. Neste mesmo ano, a planta já produz em média cinco frutos, seguido de 10 frutos no ano seguinte, e a partir do sexto ano, se estabiliza com 15 frutos. Onde o mesmo será colhido até o último ano do projeto.

Já o Paricá que inicia junto com as outras culturas, sofrerá dois desbastes com um período de tempo mais longo: o primeiro no sexto ano, com $30 \%$ do total que será colhido, e no oitavo ano o segundo desbaste com o restante da colheita que equivale a $70 \%$.

\section{ANÁLISE ECONÔMICA}

Estudos sobre viabilidade econômica de SAF têm recebido ênfase hoje em dia. Este tipo de investigação tem sempre um caráter inovador e de riqueza de informações por ser desenvolvido com um intenso aporte coletivo, haja vista, os próprios agricultores participarem como pesquisadores e reconhecedores das práticas desenvolvidas na região.

Os resultados dos indicadores de rentabilidade econômica, VPL, TIR, RB/C e PN para o SAF com paricá, mandioca e cupuaçu são apresentados na Tabela 5 . No 
que se referem à avaliação econômica, os resultados do Valor Presente Líquido (VPL) apresentados na tabela 5 mostram que o SAF apresenta viabilidade econômica, pois ao final obtém-se receita líquida atualizada positiva de $R$ \$ 13.826,61. Os resultados para o VPL obtidos na pesquisa corroboraram com os resultados de Sanguino et al., (2007), os quais obtiveram VPL positivo de R\$ 21.513,00, estudando SAF, formado com as espécies florestais, freijó e ipê-amarelo em associação com goiaba e carambola, no município de Tomé-açu (PA). Entretanto, Cordeiro et al. (2009) verificaram VPL igual -R\$ 65.971,41 em avaliação econômica de cultivo de paricá solteiro no município de Aurora do Pará (PA), mostrando que os custos foram superiores às receitas.

A Taxa Interna de Retorno (TIR) de $26 \%$ atesta a viabilidade do SAF, uma vez que a TIR foi superior à taxa de referência do mercado. Palheta et al., (2014) também obtiveram a TIR $(19,43 \%)$ acima da taxa de oportunidade, estudando SAF formado com paricá em associação com cupuaçu, feijão e mandioca no município de Santa Bárbara (PA).

Já o resultado para a Razão Benefício Custo $(\mathrm{RB} / \mathrm{C})$, indica que para cada real investido no SAF, ao final de oito anos, tem-se um retorno líquido de $R \$ 1,77$ corroborando a viabilidade do empreendimento. Os valores da RB/C também foram positivos $(6,08)$ em pesquisa realizada por Gonçalves et al. (2017), estudando a viabilidade econômica de SAF formado com paricá e açaí no assentamento Abril Vermelho no município de Santa Bárbara (PA).

De acordo com a Tabela 5 também, pode-se inferir que os resultados do Ponto de Equilíbrio (PE), para a produção do sistema agroflorestal indicam que os produtores necessitam comercializar somente $56,3 \%$ da produção para cobrir os custos. Do mesmo modo De Paula (2014) obteve um valor de PE de $41.08 \%$ analisando a viabilidade econômica do paricá em projeto de reflorestamento no município de Paragominas (PA).

TABELA 5 - Indicadores de rentabilidade econômica financeira para este SAF. Indicadores

\begin{tabular}{ll}
\hline VPL $(R \$)$ & $13.826,61$ \\
TIR & $26 \%$ \\
RB/C & 1,7766685 \\
PN & 56,285119 \\
\hline
\end{tabular}

Fonte: Dados da pesquisa

Todos os indicadores de viabilidade, VPL, Rb/c, TIR, e PN mostraram que o SAF é viável economicamente. Em função disso, recomenda-se aos produtores rurais de Santa Izabel a investirem nesses sistemas.

ANÁLISES DE SENSIBILIDADE

TABELA 6 - Aplicação de taxas para analisar a sensibilidade da receita.

\begin{tabular}{llll}
\hline Indicadores & RT (-10\%) & RT (-20\%) & RT (-30\%) \\
\hline VPL (R\$) & $10.663,70$ & $7.500,80$ & $4.337,89$ \\
\hline TIR & $26 \%$ & $26 \%$ & $26 \%$ \\
\hline RB/C & 1,5990017 & 1,421335 & 1,243668 \\
\hline PN & 62,539021 & 70,3564 & 80,40731
\end{tabular}

Fonte: Dados da pesquisa 
TABELA 7 - Aplicação de taxas para analisar a sensibilidade do custo.

$\begin{array}{llll}\text { Indicadores } & \text { CT (10\%) } & \text { CT (20\%) } & \text { CT (30\%) }\end{array}$

\begin{tabular}{llll}
\hline VPL (R\$) & $12.046,36$ & $10.266,12$ & $8.485,87$ \\
\hline TIR & $26 \%$ & $26 \%$ & $26 \%$ \\
\hline RB/C & 1,6151532 & 1,4805571 & 1,366668 \\
\hline PN & 61,913631 & 67,542143 & 73,17065 \\
\hline
\end{tabular}

Fonte: Dados da pesquisa

Foi verificado que para qualquer uma das situações analisadas, tanto de redução de receita, como de aumento de custo, haveria sustentabilidade. Porém, de acordo com os resultados, pode-se observar que o SAF é mais sensível a redução nas receita quando comparado ao aumento nos custos. Soares (2003) estudando a avaliação economica de Eucalyptus grandis destinado a multiprodutos, constataram que aumentos nas taxas de desconto e no custo da terra afetam significativamente $o$ VPL e a rentabilidade do Projeto.

\section{CONCLUSÃO}

O Sistema Agroflorestal analisado mostrou-se economicamente viável, indicando que esta atividade pode ser considerada uma forma alternativa de produção de alimento, madeira e geração de renda para os agricultores familiares locais. A análise do VPL para a produtividade, taxa de juro, receitas e custos avaliados para o projeto revelaram que o modelo considerado apresentou resultados positivos.

A análise de sensibilidade indicou que a receita é mais sensível a mudança de preços que o custo.

A adoção de Sistemas Agroflorestais que considerem o contexto da agricultura familiar no estado do Pará pode oferecer uma variedade de produtos madeireiros e não madeireiros, permitindo aos agricultores a diversificação da renda, segurança alimentar, além de recuperação ambiental de áreas.

\section{REFERÊNCIAS}

ALVES, R. M.; FILGUEIRAS, G. C.; HOMMA, A. K. O. Aspectos socioeconômicos do cupuaçuzeiro na Amazônia: do extrativismo a domesticação. In: SANTANA, A. C. (Org.). Mercado, cadeias produtivas e desenvolvimento rural na Amazônia. Belém, PA: UFRA, p.197-223. 2014.

BASA- Banco da Amazônia. PRONAF Floresta. Disponível em:<http://www.bancoamazonia.com.br/index.php/financiamentos1/floresta>. Acesso em: 24 de mai. 2018.

BERGER, R, Análise benefício-custo: instrumento de auxílio para tomada de decisões na empresa florestal, IPEF: Instituto de pesquisas e estudos florestais, circular técnica $\mathrm{n}^{\circ} \quad 97, \quad$ março/1980, Disponível em <http://www.ipef.br/publicacoes/ctecnica/nr097.pdf >.Acesso em: 24 de mai. 2018. 
BRITO, B. Regularização fundiária no Pará: afinal qual é o problema? / Brenda Brito; Dário Cardoso Junior. - Belém, PA: Instituto do Homem e Meio Ambiente da Amazônia - IMAZON, 104p. 2015.

CORDEIRO, I. M. C. C.; DE BARROS, P. L. C.; LAMEIRA, O. A.; GAZEL FILHO, A. B. Avaliação de plantios de paricá (Schizolobium parahyba var. amazonicum (Huber ex Ducke) Barneby de diferentes idades e sistemas de cultivo no Município de Aurora do Pará - PA (Brasil). Ciência Florestal, Santa Maria, v. 25, n. 3, p. 679-687, jul.-set., 2015.

CORDEIRO, I. M. C.; SANTANA, A. C.; LAMEIRAS, O. A.,; MATOS, I. S. "Economic Analysis of Farming Systems with Schizolobium parahyba var. amazonicum (Hubere $x$ Ducke) Barneby (Paricá) E Ananas Comosus var. Erectifolius (L. B. Smith) Coppus \& Leal (Curauá) in the city ofAurorado Pará (PA), Brazil." Revista de la Facultad de LUZ Agronomia. 26: 243-265. 2009.

DE PAULA, M. T.; PONTES, A. N.; FERREIRA FILHO, H. R.; CARDOSO, L. A.; SILVA, I. M.; DAMASCENO, M. C. S.; SENA, A. L. Economic Viability of Production of Tree Paricá (Schizolobium amazonicum Huber ex. Ducke) of Reforestation Project in the Municipality Paragominas-PA, Brazil. Journal of Life Sciences 8 (2014) 967971. doi: 10.17265/1934-7391/2014.12.006.

DUBOIS, J. C. L. Manual agroflorestal para a Amazônia. Rio de Janeiro: Rebraf, 1996. v. 1.

FALESI, I.C; GALEAO, R. R, Recuperação de áreas antropizadas da Mesorregião Nordeste Paraense por meio de sistemas agroflorestais. In: Congresso Brasileiro de sistemas Agroflorestais ,4., 2002, Ilhéus. Anais...Ilhéus: Sociedade Brasileira de Sistemas Agroflorestais: CEPLAC, 2004. p. 89-99.

FAO. Save and grow cassava. A guide to sustainable production intensification, 2013. Disponível em: http://www.fao.org/docrep/018/i3278e/i3278e.pdf. Acesso em: 12 mar. 2018

GONÇALVES, A. C. DA SILVA; PONTES, A. N.; DE PAULA, M. T. ; FERREIRA, P. F.; VASCONCELOS, R. C. ; FONSECA, K. O. Avaliação do perfil econômico de sistemas agroflorestais nos assentamentos dos trabalhadores rurais Expedito Ribeiro e Abril Vermelho, município de Santa Bárbara-PA. Espacio (Caracas). Vol. 38 (No 11) Año 2017. Pág. 6.

GOMES, F. T.; CARDOSO, J. H. Avaliação de dois acessos de mandioca (Manihot esculenta CRANTZ) em um sistema Agroflorestal (SAFS). XXIII Congresso de Iniciação Cientifica da Universidade Federal de Pelotas-UFPL, 2014. Disponível em: https://www.alice.cnptia.embrapa.br/bitstream/doc/1001032/1/CA03405.pdf. Acesso 13 de mar. 2018.

IBGE- Instituto Brasileiro de Geografia Estatística. Santa Izabel do Pará. Disponível em:<https://cidades.ibge.gov.br/brasil/pa/santa-izabel-do-para/panorama>. Acesso em: 13 de mar. 2018. 
KONAGANO, M; SUGAYA, C; SANTOS, D. A; MAIA E SÁ, N. Moura, M. S; SILVA, P. P. Sistema Agroflorestal de Tomé-Açu, Pará - SAFTA. In: Congresso brasileiro de sistemas agroflorestais, 10. 2016, Cuiabá - MT

PALHETA, I.C.; GOMES, C.A.S.; LOBATO, G.J.M.; PAULA, M.T.; PONTES, A.N. (2014). Viabilidade econômica de um sistema agroflorestal no município de Santa Bárbara-PA. Enciclopédia Biosfera, v. 10, n. 19, p.

PIRES, G. R.; PACHECO, J. J.; PALHETA, M. V. O.; COSTA JÚNIOR, R. N. F.; COSTA, S. V.; TOSTES, W. S. Estatísticas Municipais Santa Izabel do ParáIDESP. Disponível em: http://www.ebah.com.br/content/ABAAAe7DAAB/idespestatisticas-municipais-santa-izabel. Acessado em: 13 de mar. 2018.

PREFEITURA SANTA IZABEL, História de Santa Isabel, Disponível em < http://www.santaizabel.pa.gov.br/historia-de-santa-izabel/> Acesso em 4 jun 2016

REYDON, B.P, O desmatamento da floresta amazônica: causas e soluções, Economia verde Desafios e oportunidades, $n^{\circ} 8$, jun 2008, Disponível em < http://gestaodaterra.com.br/arquivos/O_desmatamento_da_floresta_amazonia_caus as_e_solucoes.pdf> Acesso em 1 jun 2016.

SANGUINO, A. C.; SANTANA, A. C. de.; HOMMA, A. K. O.; BARROS, P. L .C. de.; KATO, O. R.; AMIN, M. M. G. H. Análise Econômica de Investimentos em Sistemas de Produção Agroflorestal no Estado do Pará. Revista Ciência Agrária, Belém: n. 47, p. 23-47, jan. - jun. 2007.

SILVA, F.C.L.da; FARIAS, J.A.. Análise econômica da produção de Acacia mearnsii De Wild e carvão vegetal no Vale do Caí e Taquari, Rio Grande do Sul. Ciencia Rural, Santa Maria , v. 45, n. 5, p. 927-932, May 2015. Available from $<$ http://www.scielo.br/scielo.php?script=sci_arttext\&pid=S0103-

84782015000500927\&Ing=en\&nrm=iso>. Accesson 03 Apr. 2018. http://dx.doi.org/10.1590/0103-8478cr20140114.

SILVEIRA, R, Avaliação econômica da produção de madeira de Paricá (Schizolobium amazonicum Huber ex Ducke) sob diferentes espaçamentos de plantio, Jerônimo Monteiro, Espírito Santo, 2014.

SOARES, S. S., CARVALHO, R. M. M. A.; VALE, A. B. 2003. "Avaliação econômica de um povoamento de Eucalyptus grandis destinado a multiprodutos." Revista Árvore. 27: 689-694, 2003.

YAMADA, M; GHOLZ, H. L. An evaluation of agroforestry systems as a rural development option for the Brazilian Amazon. Agroforestry Systems, Netherlands, v.55, n.2, p.81-87. 2002. 